\title{
Haben wir eine moralische Pflicht zur direkten biotechnischen Lebensverlängerung?
}

\author{
Jakob Lohmar
}

Online publiziert: 28 . Oktober 2020

(C) Der/die Autor(en) 2020

Zusammenfassung Wenn eine Person unter einer tödlichen Krankheit leidet und nicht über die Ressourcen für eine medizinische Behandlung verfügt, sind wir normalerweise dazu verpflichtet, ihr die notwendigen Ressourcen bereitzustellen. Wären wir aber in einem biotechnischen Zukunftsszenario, in dem die menschliche Lebensspanne durch Eingriffe in den Alterungsprozess erhöht werden kann, auch dazu verpflichtet, anderen Personen die notwendigen Ressourcen für solche Maßnahmen bereitzustellen? John Harris hat argumentiert, dass wir zu solch einer direkten biotechnischen Lebensverlängerung verpflichtet wären, da ein Leben zu verlängern das Gleiche sei, wie ein Leben zu retten. Im vorliegenden Aufsatz wird gezeigt, dass Harris' Argument nicht schlüssig ist, da entgegen seiner Prämisse ein begrifflicher Unterschied zwischen der Rettung und der Verlängerung eines Lebens besteht. Auf Grundlage von Harris' zurückgewiesenem Argument werden dann aber zwei alternative Argumente für eine (prima facie) Pflicht zur direkten Lebensverlängerung konstruiert. Das erste Argument basiert auf der Überlegung, dass durch eine direkte biotechnische Lebensverlängerung trotz des begrifflichen Unterschieds mit einer substanziellen Wahrscheinlichkeit Leben gerettet werden würden. Das zweite Argument besagt, dass trotz deskriptiver Unterschiede eine moralische Äquivalenz zwischen der Rettung und der Verlängerung eines Lebens besteht, sofern durch die bloße Lebensverlängerung das Leben um die gleiche Dauer und Qualität verlängert wird wie durch die Lebensrettung.

Schlüsselwörter Anti-Aging · Lebensverlängerung · Lebensrettung · Moralische Pflichten · Biotechnologie

J. Lohmar $(\bowtie)$

Philosophisches Seminar, Universität zu Köln, Albertus-Magnus-Platz, 50923 Köln, Deutschland

E-Mail: JakobLohmar@gmx.de 
Abstract When a person suffers from a deadly disease and is lacking the resources for a medical treatment, we are normally obliged to provide the necessary resources. But would we also be morally obliged to provide the resources for an extension of someone's lifespan through anti-aging treatments which might be developed due to biotechnological progress? John Harris has argued that there would be an obligation to such a direct biotechnical life extension because extending a life is the same as saving a life. In the present paper, it will be shown that Harris' argument is not sound since, contrary to his premise, there is a conceptual difference between extending and saving a life. On the basis of Harris' rejected argument, though, two alternative arguments for a (prima facie) obligation to direct biotechnical life extension will be developed. The first argument is based on the idea that, despite the conceptual difference, one saves someone's life with a substantial probability when extending it by direct biotechnical means. In the second argument, it will be argued that there is a moral equivalence between extending and saving someone's life despite the descriptive differences as far as the additional lifetime has the same duration and quality in both cases.

Keywords Anti-Aging - Life Extension - Lifesaving · Moral Obligations · Biotechnology

\section{Einleitung}

Momentan gibt es noch keine bewährte Methode, um die menschliche Lebenserwartung durch Eingriffe in den biologischen Alterungsprozess deutlich zu erhöhen. Inzwischen wird jedoch aktiv und mit ersten (umstrittenen) Erfolgen an der Entwicklung solcher Methoden geforscht; ${ }^{1}$ die Biogerontologie hat Fortschritte im Verständnis der kausalen Prozesse, die dem biologischen Altern zu Grunde liegen, erzielt; und viele Hypothesen wurden darüber aufgestellt, wie ein Eingriff in den menschlichen Alterungsprozess durch verschiedene Biotechnologien erreicht werden könnte (s. z.B. Gems 2009, Rose 2009 und Freitas 2009). Wie wahrscheinlich die Entwicklung einer effektiven Anti-Aging-Technologie ist und wann diese (wenn überhaupt) zu erwarten ist, wird unter Experten äußerst kontrovers diskutiert (s. ebd.). Anstatt darauf zu spekulieren, dass es nicht oder erst in der fernen Zukunft zu der Entwicklung einer Anti-Aging-Technologie kommen wird (und später eventuellen technologischen Entwicklungen hinterherzuhinken), haben sich viele Ethiker bereits mit der ethischen Beurteilung solch eines biotechnischen Szenarios befasst. Der vorliegende Aufsatz soll einen Beitrag zu dieser Debatte leisten.

Die genaue ethische Frage, der in diesem Aufsatz nachgegangen wird, kann wie folgt eingeführt werden: Wenn eine Person unter einer tödlichen Krankheit leidet und nicht über die Ressourcen für eine medizinische Behandlung verfügt, sind wir

\footnotetext{
1 Wie Bostrom (2005: 276) aufführt, konnten z. B. durch Kalorienrestriktion altersbedingte Krankheiten verschiedener Tierarten aufgeschoben und ihre Lebensspanne deutlich verlängert werden. Neuere Forschungsansätze halten den Wirkstoff Metformin, dessen Effekte auf den Alterungsprozess momentan getestet werden, sogar in Anwendung auf den Menschen für vielversprechend (s. Barzilai et al. 2016).
} 
normalerweise dazu verpflichtet, ihr die notwendigen Ressourcen bereitzustellen. Wären wir aber in einem biotechnischen Zukunftsszenario, in dem die Lebenserwartung durch eine Verlangsamung des Alterungsprozesses erhöht werden kann, auch dazu verpflichtet, anderen Personen die notwendigen Ressourcen für solche Maßnahmen bereitzustellen? Während klar ist, dass eine gewisse moralische Verpflichtung besteht, das Leben anderer Personen durch die Heilung tödlicher Krankheiten auf indirektem Wege zu verlängern, ist fraglich, ob wir ebenfalls zu einer direkten Lebensverlängerung durch Eingriffe in den Alterungsprozess verpflichtet wären. ${ }^{2}$

In der philosophischen Literatur sind bereits zahlreiche moralische Gesichtspunkte gegen die direkte Lebensverlängerung hervorgebracht und diskutiert worden. Dazu zählen z.B. populationsethische Bedenken, die direkte Lebensverlängerung würde zu Überbevölkerung oder aber einer (pro Einheit Lebenszeit) weniger glücklichen Bevölkerung führen (s. Singer 2009), sowie verschiedene Gerechtigkeitsprobleme wie z. B. Unfairness gegenüber denjenigen, die nicht von der direkten Lebensverlängerung profitieren würden (s. Harris 2007: 62-63), oder besonders schlecht gestellten Gruppen wie Menschen, die bereits in ihrer Jugend an tödlichen Krankheiten erkranken (s. Farrelly 2008: 263-66), oder aber Menschen, die unter den Folgen extremer Armut leiden (s. Chapman 2004). Um feststellen zu können, ob diese Gesichtspunkte letztlich in der Frage, ob wir all things considered zur direkten Lebensverlängerung verpflichtet wären, entscheidend sind, ist es nötig zu wissen, wie starke moralische Gründe überhaupt zum direkten Verlängern eines menschlichen Lebens bestehen. Es muss also in Abstraktion aller positiven und negativen Effekte (konkreter Umsetzungen) der direkten Lebensverlängerung bestimmt werden, welchen moralischen Status die direkte Lebensverlängerung per se hat. ${ }^{3}$

Ich werde in diesem Aufsatz dieser Frage nachgehen, indem ich ein Argument von John Harris (2007) prüfe, nach welchem wir eine moralische Pflicht zur direkten Lebensverlängerung haben, welche moralisch äquivalent zu unserer Pflicht zur Lebensrettung ist. Bei dieser Prüfung werde ich mich auf Harris' begriffliche Prämisse konzentrieren, nach der ein Leben zu retten das Gleiche ist, wie ein Leben zu verlängern, indem ich mich mit Héctor Wittwers (2009) Kritik an dieser Prämisse befasse und eigene begriffliche Überlegungen anstelle. Nachdem die Prüfung von Harris' Argument Gegenstand des folgenden zweiten Teils dieses Aufsatzes gewesen sein wird, werde ich auf dieser Basis im dritten Teil zwei weitere Argumente dafür entwickeln, dass wir eine moralische Pflicht zur direkten Lebensverlängerung haben. Im abschließenden vierten Teil werden die wichtigsten Ergebnisse des Aufsatzes zusammengefasst und ihre Relevanz beurteilt.

\footnotetext{
2 Die Terminologie von direkter und indirekter Lebensverlängerung habe ich von Wittwer (2009) übernommen. „Direkte Lebensverlängerung“ werde ich im Folgenden einfach als Abkürzung für „Lebensverlängerung durch einen Eingriff in den Alterungsprozess“ verwenden. Das soll nur der besseren Leserlichkeit dienen; eine philosophische oder medizinische Behauptung soll hierdurch nicht impliziert werden.

3 Um ganz präzise zu sein, müsste die Frage wohl wie folgt formuliert werden: Wie starke moralische Gründe gibt uns die (für das Vorliegen einer direkten Lebensverlängerung notwendige und hinreichende) Eigenschaft einer direkten Lebensverlängerung, dass durch sie das Leben einer Person auf direktem Wege verlängert wird?
} 


\section{Harris' Argument für eine moralische Pflicht zur direkten Lebensverlängerung}

Harris stellt sein Argument dafür, dass wir eine Pflicht zur direkten Lebensverlängerung haben, wie folgt dar:

When we save a life, by whatever means, we simply postpone death. Since lifesaving is just death-postponing with a positive spin, it follows that life-extending therapies are, and must always be, life-saving therapies and must share whatever priority lifesaving has in our morality and in our social values. So long as the life is of acceptable quality (acceptable to the person whose life it is) we have a powerful - and many would claim overriding - moral imperative to save the life, because to fail to do so when we can would make us responsible for the resulting death (Harris 2007: 61).

Harris behauptet also zunächst, dass ein Leben zu retten nichts anderes ist, als den Tod einer Person aufzuschieben, und somit das Gleiche ist, wie ein Leben zu verlängern. Daraus folgert er, dass ein Leben zu verlängern auch den gleichen moralischen Status haben muss, wie ein Leben zu retten. Die weitere von Harris nicht explizierte Prämisse, die erforderlich ist, damit letzteres logisch folgt, ist die, dass zwei Dinge den gleichen moralischen Status haben, wenn sie (in deskriptiver Hinsicht) das Gleiche sind. ${ }^{4}$ Nachdem Harris so für die moralische Äquivalenz von Lebensrettung und Lebensverlängerung argumentiert hat, spezifiziert er den moralischen Status einer Lebensrettung, indem er behauptet, dass wir eine starke Pflicht dazu haben, sofern das Leben von akzeptabler Qualität ist. Daraus, dass er diese Pflicht als stark (,,powerful“") charakterisiert, geht hervor, dass er die Pflicht zur Lebensrettung nicht als absolute Pflicht einschätzt, sondern nur als eine - obgleich starke - prima facie Pflicht, die bei einem Pflichtenkonflikt durch eine andere Pflicht übertrumpft werden könnte. ${ }^{5}$ Der naheliegende Schluss, den Harris selbst nicht mehr expliziert, ist der, dass wir entsprechend auch eine (starke) prima facie Pflicht zur Lebensverlängerung haben, sofern das Leben eine akzeptable Qualität hat. Da Harris letztlich dafür argumentieren möchte, dass wir eine prima facie Pflicht zur direkten Lebensverlängerung haben, sollte es in seinem Sinne sein, das Argument um die Prämisse zu ergänzen, dass, wenn wir eine prima facie Pflicht zur Lebensverlängerung haben, wir auch eine prima facie Pflicht zur direkten Lebensverlängerung haben, sodass geschlossen werden kann, dass wir eine prima facie Pflicht zur direkten Lebensverlängerung haben, sofern das verlängerte Leben von akzeptabler Qualität ist.

\footnotetext{
4 Natürlich folgt daraus, dass sie sowohl in deskriptiver als auch normativer Hinsicht gleich sind, dass sie in normativer Hinsicht gleich sind und damit den gleichen moralischen Status haben, sodass dann keine zusätzliche Prämisse benötigt werden würde. Harris will aber in der ersten Prämisse noch keine normative Aussage, sondern eine rein deskriptive Aussage über das begriffliche Verhältnis von Lebensrettung und Lebensverlängerung machen. Dann wird aber die oben genannte weitere Prämisse für einen gültigen Schluss benötigt.

5 Die Anmerkung, dass viele die Pflicht zur Lebensrettung sogar als ,overriding“ einschätzen, lässt vermuten, dass Harris auch nicht ausschließen will, dass es sich um eine absolute Pflicht handelt. Sein Argument kann dann so interpretiert werden, dass zumindest eine prima facie Pflicht besteht.
} 
Das so erweiterte und vollständig explizierte Argument kann dann wie folgt dargestellt werden:

(H1) Lebensverlängerung und Lebensrettung sind in deskriptiver Hinsicht das Gleiche.

(H2) Was in deskriptiver Hinsicht gleich ist, hat den gleichen moralischen Status.

(K1) Lebensverlängerung und Lebensrettung haben den gleichen moralischen Status (aus $\mathrm{H} 1$ und $\mathrm{H} 2$ ).

(H3) Lebensrettung hat den moralischen Status einer prima facie Pflicht, sofern sie sich auf Leben mit akzeptabler Qualität bezieht.

(K2) Lebensverlängerung hat den moralischen Status einer prima facie Pflicht, sofern sie sich auf Leben mit akzeptabler Qualität bezieht (aus K1 und H3).

(H4) Wenn wir eine prima facie Pflicht zur Lebensverlängerung haben, haben wir auch eine prima facie Pflicht zur direkten Lebensverlängerung.

(K3) Wir haben eine prima facie Pflicht zur direkten Lebensverlängerung, sofern sie sich auf Leben mit akzeptabler Qualität bezieht (aus K2 und H4).

Wie man an dieser Darstellung erkennen kann, basiert Harris' Argument auf den vier Prämissen (H1) bis (H4), während es sich bei (K1) bis (K3) um Schlussfolgerungen aus diesen Prämissen handelt. Während die Prämisse H1, in der die deskriptive Gleichheit von Lebensrettung und Lebensverlängerung behauptet wird, zunächst eher unplausibel oder zumindest überraschend ist, sind die übrigen Prämissen H2 bis $\mathrm{H} 4$ auf den ersten Blick sehr plausibel und könnten sogar als selbstverständlich erachtet werden (was wahrscheinlich auch der Grund ist, weshalb Harris sie zum Teil nicht expliziert). Bevor ich die strittige Prämisse H1 eingehend untersuche, werde ich aber zunächst noch einen zweiten Blick auf die übrigen Prämissen werfen, um zu prüfen, ob sie tatsächlich so selbstverständlich sind, wie sie auf den ersten Blick scheinen.

\subsection{Supervenienz moralischer Eigenschaften}

Die Prämisse H2 besagt, dass Dinge, die in deskriptiver Hinsicht gleich sind, auch den gleichen moralischen Status haben. Alternativ könnte man die Prämisse auch so formulieren: Was die gleichen natürlichen Eigenschaften hat, hat auch die gleichen moralischen Eigenschaften. Diese Aussage folgt unmittelbar aus der Supervenienz moralischer Eigenschaften - oder allgemeiner: normativer Eigenschaften - auf natürlichen Eigenschaften, welche weitestgehend anerkannt ist (s. Lutz und Lenman 2018). Tatsächlich müsste für die Gültigkeit des Arguments nicht einmal behauptet werden, dass für alle Dinge gilt, dass sie den gleichen moralischen Status haben, wenn sie sich in deskriptiver Hinsicht gleichen, sondern nur, dass dies in Bezug auf Lebensrettung und Lebensverlängerung gilt. Und auch das ist höchst plausibel: 
Wenn es in deskriptiver Hinsicht keinen Unterschied zwischen Lebensrettung und Lebensverlängerung gibt, so haben sie sicherlich auch den gleichen moralischen Status.

\subsection{Der moralische Status einer Lebensrettung}

Die Prämisse H3 besagt, dass wir prima facie verpflichtet sind, Leben von akzeptabler Qualität zu retten. Es ist klar, dass wir in den meisten Fällen prima facie zur Lebensrettung verpflichtet sind. Fraglich ist nur, welche Ausnahmen es gibt, in denen diese prima facie Pflicht nicht besteht. ${ }^{6}$ Harris (2007: 61) zufolge besteht eine solche Ausnahme dann, wenn das gerettete Leben nicht von akzeptabler Qualität wäre. In der Tat scheint es für den moralischen Status einer Lebensrettung zumindest große Relevanz zu haben, ob das gerettete Leben überhaupt lebenswert wäre. Unabhängig davon könnte es aber auch relevant sein, ob die Person, um deren Leben es sich handelt, (nach reichlicher Überlegung und bei vollständiger Information) weiterleben will oder ob sie ihren Tod vorzieht (s. Knell 2011: 13). Schließlich könnte auch von Relevanz sein, wie viel zusätzliche Lebenszeit man der geretteten Person durch die Lebensrettung beschert. Wie Wittwer (2009: 223) betont, ist es mit einer Lebensrettung vereinbar, dass die Person kurze Zeit danach an einer anderen Todesursache stirbt. Es könnte nun sein, dass keine prima facie Pflicht zur Lebensrettung besteht, wenn man den Todeszeitpunkt dadurch nur minimal - zum Beispiel um nur wenige Minuten oder gar Sekunden - nach hinten verschiebt.

Es schließt sich die schwierige Frage an, welche Kombinationen dieser potentiell moralisch relevanten Faktoren dazu führen, dass keine prima facie Pflicht zur Lebensrettung besteht. Dieser Frage kann hier nicht nachgegangen werden. Unabhängig davon, wie sie zu beantworten ist, kann jedoch festgehalten werden, dass wir zumindest dann eine prima facie Pflicht zur Lebensrettung haben, wenn keiner der drei potentiellen Ausnahmefälle auftritt - wenn also die gerettete Person ihre Lebensrettung will und durch sie ihr Leben mehr als nur minimal und durch lebenswerte Lebenszeit verlängert wird. Aus Harris' Argument würde sich also zumindest ergeben, dass wir prima facie verpflichtet sind, das Leben von solchen Personen (direkt) zu verlängern, die diese Lebensverlängerung wollen und bei denen zu erwarten ist, dass sich ihr Leben mehr als nur minimal und durch lebenswerte Lebenszeit verlängern wird. Diese Konklusion wäre aber aus philosophischer Sicht kaum weniger interessant als die ursprüngliche. (Dass Harris mit seinem Argument den moralischen Status aller moralisch besonders problematischen Fälle von direkter Lebensverlängerung klären kann, war ohnehin nicht zu erwarten.) Es bliebe dabei, dass direkte Lebensverlängerung den gleichen moralischen Status wie Lebensrettung hat, sodass in vielen hypothetischen Fällen eine prima facie Pflicht zur direkten Lebensverlängerung bestehen würde.

\footnotetext{
6 Die Frage ist hier nicht, welche besonderen Umstände es geben könnte, sodass wir all things considered nicht zur Lebensrettung verpflichtet sind, sondern die Frage ist, welche Fälle es gibt, in denen wir nicht einmal prima facie zur Lebensrettung verpflichtet sind - in denen die Rettung eines Lebens uns also nicht einmal einen moralischen Grund zum Handeln gibt, der prinzipiell stark genug ist, um uns zu der Lebensrettung (all things considered) zu verpflichten.
} 
Andererseits könnten sich in praktischer Hinsicht durchaus größere Einschränkungen der prima facie Pflicht zur direkten Lebensverlängerung ergeben. Das könnte zum Beispiel der Fall sein, wenn die ersten technologischen Realisierungen der direkten Lebensverlängerung noch keine großen Auswirkungen auf die Lebenserwartung haben würden, oder wenn in der Bevölkerung Skepsis gegenüber Eingriffen in den Alterungsprozess bestünde, sodass diesen oft nicht zugestimmt werden würde. Die Einschränkung, dass nur lebenswertes Leben verlängert werden muss, sollte in praktischer Hinsicht aber nur wenig relevant sein, sofern die Verlängerung der Lebenszeit keine gigantischen, unüberschaubaren Ausmaße annimmt. Denn selbst wenn Kritiker der direkten Lebensverlängerung wie Bernard Williams (1973: 81100) damit recht haben sollten, dass die Qualität zusätzlicher Lebenszeit ab einem gewissen hohen Alter abnehmen würde, so ist es doch sehr unplausibel, dass nicht nur die Qualität pro Lebensjahr, sondern auch die Gesamtqualität des Lebens durch zusätzliche Lebensjahre sinken würde. Die Unplausibilität dieser Behauptung ergibt sich insbesondere vor dem Hintergrund, dass es das Ziel der direkten Lebensverlängerung ist, die Lebensspanne um gesunde und vitale Jahre zu verlängern (s. Farrelly 2008: 261). Doch selbst wenn auch die Gesamtqualität des Lebens überraschenderweise sinken sollte, sind wir doch weit davon entfernt, dies im Vorhinein wissen zu können. Wenn Harris damit recht hat, dass direkte Lebensverlängerung und Lebensrettung moralisch äquivalent sind, so ist der einzige moralisch zulässige Umgang mit dieser epistemischen Unsicherheit, die Lebensverlängerung durchzuführen. Denn auch wenn wir uns nicht darin sicher sein können, ob eine sich in Lebensgefahr befindliche Person ein lebenswertes Leben haben wird, wenn wir sie retten - worin wir uns bei fremden Personen quasi nie sicher sein können -, sind wir prima facie verpflichtet sie zu retten. Entsprechendes muss dann auch für die direkte Lebensverlängerung gelten, wenn moralische Äquivalenz zwischen Lebensrettung und -verlängerung besteht. ${ }^{7}$

Zusammenfassend lässt sich über die Prämisse H3 sagen, dass es neben der Qualität des geretteten Lebens vermutlich noch andere Faktoren gibt, die darüber entscheiden, ob eine prima facie Pflicht zur Lebensrettung besteht, sodass es - entgegen H3 - sein könnte, dass in Ausnahmefällen auch dann keine prima facie Pflicht zur Lebensrettung besteht, wenn es sich um Leben mit akzeptabler Qualität handelt. Die Prämisse H3 und die Konklusion können aber leicht angepasst werden, ohne dass dabei viel philosophischer Gehalt verloren geht. Die Frage, welche der potentiellen Ausnahmefälle in welchem Maße auftreten werden, ist zu diesem Zeitpunkt noch sehr spekulativ. Es konnte aber ein Argument dafür entwickelt werden, dass uns die spekulative Befürchtung, zusätzliche Lebenszeit könnte nicht lebenswert sein, nicht von der prima facie Pflicht zur direkten Lebensverlängerung befreien würde, sollte es eine solche Pflicht überhaupt geben.

\footnotetext{
7 Siehe Schloendorn (2006: 195) für eine konsequentialistische Argumentation dafür, dass wir auch im Fall epistemischer Unsicherheit über die zu erwartende Lebensqualität zur Lebensverlängerung verpflichtet sind.
} 


\subsection{Unnatürlichkeit der direkten Lebensverlängerung}

H4 - die letzte der auf den ersten Blick plausiblen Prämissen - besagt, dass wir eine prima facie Pflicht zur direkten Lebensverlängerung haben, wenn wir eine prima facie Pflicht zur Lebensverlängerung haben. Dass dies der Fall ist, ist keine logische Notwendigkeit: Es ist logisch möglich, dass wir eine prima facie Pflicht zur Lebensverlängerung, aber nicht zur direkten Lebensverlängerung haben. Dafür müsste es starke moralische Gründe geben, die spezifisch dagegen sprechen, Leben auf direkte Weise - also durch Eingriffe in den Alterungsprozess - zu verlängern. Könnte ein Eingriff in den biologischen Alterungsprozess eines Menschen moralisch problematisch sein? Es könnte die Sorge aufkommen, dass ein solcher Eingriff unnatürlich ist (s. Kass 2003: 20). Ein Eingriff in den biologischen Alterungsprozess scheint aber nicht weniger natürlich zu sein als alle möglichen anderen medizinischen Eingriffe in die menschliche Konstitution, die ethisch unstrittig sind (s. ebd.: 21). Dazu zählen insbesondere Maßnahmen der indirekten Lebensverlängerung, von denen auch Skeptiker der moralischen Notwendigkeit der direkten Lebensverlängerung meinen, dass sie zweifellos moralisch geboten sind (s. z.B. Wittwer 2009: 215). Dann sollte aber auch in Bezug auf die direkte Lebensverlängerung gelten, dass ihre vermeintliche Unnatürlichkeit keinen Grund darstellt, an ihrer (eventuellen) moralischen Notwendigkeit zu zweifeln. Entweder sind medizinische Maßnahmen wie die der direkten und indirekten Lebensverlängerung gar nicht so „unnatürlich“ oder ihre Unnatürlichkeit ändert nichts an ihrer moralischen Notwendigkeit. ${ }^{8}$

\subsection{Die Begriffe der Lebensverlängerung und der Lebensrettung}

Bisher wurden die Prämissen H2 bis H4 von Harris' Argument geprüft. Die anfängliche Plausibilität dieser Prämissen konnte dabei grundsätzlich bestätigt werden. Im Folgenden werde ich die verbleibende Prämisse H1 untersuchen, die im Gegensatz zu den anderen Prämissen intuitiv fraglich erscheint.

Die Prämisse H1 besagt, dass ein Leben zu retten nichts anderes ist, als ein Leben zu verlängern. Diese Aussage wirkt zunächst überraschend: Sicherlich verlängern wir ein Leben (zumindest minimal), wenn wir es retten, aber tun wir, indem wir es retten, auch nichts anderes, als es zu verlängern? Gibt es nicht auch Fälle, in denen ein Leben verlängert wird, ohne dass es gerettet wird? Héctor Wittwer (2009) hat in Antwort auf Harris' Argument eine Analyse unseres Begriffs der Lebensrettung durchgeführt und ist zu dem Resultat gelangt, dass es neben der Lebensverlängerung noch mehrere andere notwendige Bedingungen für das Vorliegen einer Lebensrettung gibt. Wenn er damit recht hat, sind Lebensverlängerung und Lebensrettung nicht das Gleiche, sodass die Prämisse H1 falsch und Harris' Argument somit nicht schlüssig wäre.

Die erste von Wittwer (2009: 223) vorgeschlagene zusätzliche notwendige Bedingung ist, dass ein gerettetes Leben akut gefährdet gewesen sein muss. Somit könne

\footnotetext{
8 Arthur Caplan (2004) konstatiert, dass Altern (in einer bestimmten Geschwindigkeit) kein intrinsisches Merkmal der menschlichen Natur ist. Auf dieser Grundlage könnte argumentiert werden, dass eine Verlangsamung des Alterns (in einem tieferen Sinne) gar nicht unnatürlich ist.
} 
man nicht von einer Lebensrettung sprechen, wenn die Person ohne die Rettung nur „,irgendwann“ gestorben wäre. Diese Erläuterung legt die Interpretation nahe, dass es nach Wittwer notwendig ist, dass die Person schon relativ kurze Zeit nach der Rettung gestorben wäre. Das scheint jedoch nicht der Fall zu sein: Es würde sich um eine Lebensrettung handeln, wenn einer vergifteten Person ein Antidot gegeben wird, auch wenn das Gift sie erst zu einem bedeutend späteren Zeitpunkt - wie z. B. erst nach Wochen oder sogar erst nach einem Jahr - getötet hätte. ${ }^{9}$ Allerdings ist die Todesgefahr in diesem hypothetischen Beispiel in einem anderen Sinne durchaus akut: Das Gift, das den Tod letztlich verursacht hätte, ist zum Zeitpunkt der Rettung bereits kausal wirksam. Vielleicht muss ein Leben in dem Sinne akut bedroht sein, dass die kontrafaktische direkte Todesursache (also das, was den Tod letztlich ohne die Rettung verursacht hätte) bereits kausal wirksam ist, damit man das Leben retten kann? Auch das scheint nicht zuzutreffen: Es handelt sich um eine Lebensrettung, wenn man eine zum Tode verurteilte Person aus dem Gefängnis befreit, sodass diese fliehen kann. Zu diesem Zeitpunkt ist aber die kontrafaktische direkte Todesursache, welche in der geplanten Art der Hinrichtung besteht, noch in keiner Weise wirksam. ${ }^{10}$

Die zweite von Wittwer (ebd.) vorgeschlagene notwendige Bedingung ist, dass es für mindestens einen Menschen klar erkennbar gewesen sein muss, dass sich die gerettete Person in Lebensgefahr befand. Dass es sich hierbei um keine notwendige Bedingung handelt, zeigen die folgenden Fälle: Auch wenn niemand mit Sicherheit wissen konnte, dass eine vergiftete Person an dem Gift gestorben wäre, handelt es sich um eine Lebensrettung, wenn die Person de facto daran gestorben wäre. Ebenso handelt es sich um eine Lebensrettung, wenn man einen Nichtschwimmer aus einem See zieht, wenn dieser de facto ohne den Eingriff gestorben wäre, auch wenn dies für alle unklar war, da niemand ausschließen konnte, dass er sich selbst aus dem Wasser hätte kämpfen können. Es kommt also nur darauf an, dass die Person ohne die Rettung tatsächlich gestorben wäre und nicht darauf, dass man dies wusste. Natürlich kann man in dem Fall, in dem man das nicht weiß, auch nicht mit Sicherheit behaupten, dass man die Person gerettet hat. Aber das liegt daran, dass man nicht weiß, ob eine notwendige Bedingung für eine Lebensrettung - das Vorliegen einer Todesgefahr - erfüllt ist, und nicht daran, dass dies zu wissen selbst eine notwendige Bedingung ist. Wenn es sich dabei um eine notwendige Bedingung handelte, wäre die Konsequenz nämlich nicht, dass man nicht weiß, ob man eine Person gerettet hat, wenn man nicht weiß, ob sie gestorben wäre, sondern die Konsequenz wäre, dass man sie mit Sicherheit nicht gerettet hat, da dann ja eine notwendige Bedingung für eine Lebensrettung unerfüllt wäre. Ebenso wie es für das Vorliegen eines Junggesellen nicht notwendig ist, dass man weiß, dass

\footnotetext{
9 Hans-Jörg Ehni (2013: 255) führt als weitere - allerdings, wie ich finde, intuitiv weniger klare - Gegenbeispiele Rauchverbote und Vorsorgeuntersuchungen auf. Auch hierbei handle es sich um lebensrettende Maßnahmen, obgleich die geretteten Leben nicht unmittelbar bedroht seien.

10 Natürlich unterbricht eine Lebensrettung irgendein Element der Kausalkette, die zum Tod der geretteten Person geführt hätte (wie im Beispiel die Gefangenschaft). Gleiches gilt aber auch für eine Lebensverlängerung. Siehe dazu meine Diskussion von Wittwers Bedingung (ii).
} 
er unverheiratet ist, ist es daher auch nicht für das Vorliegen einer Lebensrettung notwendig, dass man wei $\beta$, dass eine Todesgefahr bestand.

Die nächste von Wittwer (ebd. unter ,(ii)“) vorgeschlagene notwendige Bedingung ist, dass die Lebensrettung das Überleben des Geretteten kausal bewirkt haben muss. Hingegen sei es im Fall von altersverzögernden Eingriffen schwierig festzustellen, ob sie tatsächlich bewirkt haben, dass die Person länger gelebt hat, da dies auch auf andere kausale Faktoren zurückgeführt werden könnte. Es ist offensichtlich richtig, dass eine Lebensrettung kausal wirksam gewesen sein muss. Das Gleiche gilt aber auch für eine (direkte) Lebensverlängerung: Es ist für eine (direkte) Lebensverlängerung nicht hinreichend, dass man mit einer Person interagiert und diese daraufhin länger lebt. Die Interaktion mit dieser Person muss bewirkt haben, dass sie länger lebt; eine bloße Korrelation ist nicht hinreichend. Das vermeintliche epistemische Problem, im Einzelfall festzustellen, ob ein alternsverzögernder Eingriff für ein langes Leben ursächlich war oder nur damit korreliert, ändert an dieser begrifflichen Tatsache nichts. ${ }^{11}$

Schließlich argumentiert Wittwer (ebd. unter ,(iii)“) dafür, dass es für das Vorliegen einer Lebensrettung irrelevant ist, wie lange die gerettete Person nach ihrer Lebensrettung weiterlebt, während eine direkte Lebensverlängerung nur vorliege, wenn die Person überdurchschnittlich lang lebt. Mir scheint, dass eine direkte Lebensverlängerung auch dann vorliegen kann, wenn die Person nicht überdurchschnittlich lang lebt. So handelt es sich z. B. um eine direkte Lebensverlängerung, wenn eine altersbedingte tödliche Krankheit durch eine Verlangsamung des Alterungsprozesses verhindert wird, auch wenn die Person kurz danach an einem Autounfall stirbt, sodass sie nicht überdurchschnittlich lang lebt. In jedem Fall - also auch wenn dies für eine direkte Lebensverlängerung notwendig sein sollte - besteht hierin aber keine notwendige Bedingung für eine Lebensverlängerung. Für diese ist nur notwendig, dass die Person länger lebt, als sie ohne die Lebensverlängerung gelebt hätte, nicht aber, dass sie überdurchschnittlich lang lebt. Es ergibt sich also kein Unterschied zwischen Lebensrettung und Lebensverlängerung - und nur darum geht es in der hier diskutierten Prämisse H1. ${ }^{12}$

Obwohl sich auf Basis von Wittwers Begriffsanalyse kein Unterschied zwischen Lebensrettung und Lebensverlängerung feststellen ließ, denke ich, dass es einen begrifflichen Unterschied gibt. Wittwer (ebd.: 223-24) hat richtig festgestellt, dass es sich auch dann um eine Lebensrettung handelt, wenn man einen Nichtschwimmer vor dem Ertrinken bewahrt, wenn dieser zwei Stunden danach in einem Autounfall ums Leben kommt. In diesem Fall treten also sowohl Lebensrettung als auch Le-

\footnotetext{
11 Abgesehen davon sollte es nicht besonders schwierig sein diese Kausalität festzustellen, wenn - wie gewöhnlich in der ethischen Diskussion angenommen wird (s. z. B. Singer 2009) - die direkte Lebensverlängerung zu einer beträchtlichen Verlängerung (wie einer Verdoppelung) der Lebenszeit führt. Noch einfacher sollte es dadurch werden, dass vermutlich nicht alle Menschen gleichzeitig Zugang zu alternsverzögernden Eingriffen erhalten würden, sodass es quasi eine Kontrollgruppe gäbe.

12 Dass Lebensrettung und direkte Lebensverlängerung nicht das Gleiche sind, ist ohnehin klar. Fraglich ist nur, ob es sich bei der direkten Lebensverlängerung um eine Art der Lebensrettung handelt (was der Fall ist, wenn Lebensverlängerung und Lebensrettung das Gleiche sind). Dass wir eine prima facie Pflicht zur direkten Lebensverlängerung haben, wenn wir eine Pflicht zur Lebensverlängerung haben, wurde bereits unter der Prämisse H4 diskutiert.
} 
bensverlängerung auf. Aber was wäre, wenn es einem nur gelingt, dem Ertrinkenden ein Stück Holz zuzuwerfen, auf dem er sich noch zwei Stunden lang über Wasser halten kann, bevor er ertrinkt? Man hätte sein Leben ebenso wie im ersten Fall um zwei Stunden verlängert; aber hätte man sein Leben auch gerettet? Das scheint nicht der Fall zu sein. Der Grund, weshalb man hier nicht von einer Lebensrettung sprechen kann, besteht darin, dass die lebensbedrohliche Gefahr nicht vollständig abgewendet, sondern ihre tödliche Wirkung nur hinausgezögert wurde. Demnach besteht ein begrifflicher Unterschied zwischen Lebensrettung und Lebensverlängerung darin, dass es für eine Lebensrettung notwendig ist, dass das gerettete Leben verlängert wurde, indem eine Todesgefahr vollständig abgewendet wurde, während es für eine Lebensverlängerung irrelevant ist, auf welche Weise das Leben verlängert wurde, sodass es auch hinreicht, die tödliche Wirkung einer Todesgefahr aufzuschieben. Dies lässt sich an einem weiteren Beispiel illustrieren: Wenn man die tödlichen Verletzungen einer verunglückten Person heilt, rettet man ihr Leben, auch wenn sie einen Tag später an einer von diesen Verletzungen unabhängigen Todesursache stirbt. Wenn man aber das Leben dieser Person um einen Tag verlängert, indem man ihre Verletzungen medizinisch behandelt, sie aber trotzdem nach einem Tag an diesen Verletzungen stirbt, verlängert man zwar ihr Leben, rettet es aber nicht.

Was ist aber, wenn die tödliche Wirkung einer Todesgefahr um sehr lange Zeit hinausgezögert wird? Colin Farrelly (2008: 260) führt z. B. den hypothetischen Fall an, dass der tödliche Effekt einer Krankheit, an der ein zweijähriges Kind erkrankt ist, durch eine medizinische Intervention um 20 oder sogar 40, 70 oder 100 Jahre hinausgezögert wird. Handelt es sich selbst dann nicht um eine Lebensrettung? Ich habe hierzu keine klare Intuition, und ähnlich scheint es auch Farrelly zu gehen, da er die Frage unbeantwortet lässt. Auch ohne diese schwierige Frage zu beantworten, ${ }^{13}$ lässt sich aber in jedem Fall Folgendes festhalten: Es ist für das Vorliegen einer Lebensrettung hinreichend, dass das Leben einer Person verlängert wird, indem eine Gefahr, die ihr Leben bedroht (und die sie de facto getötet hätte), vollständig abgewendet wird. Vielleicht ist dies für eine Lebensrettung zudem notwendig. Vielleicht ist es aber für eine Lebensrettung daneben auch hinreichend, dass das Leben einer Person verlängert wird, indem die tödliche Wirkung einer Lebensgefahr um lange Zeit aufgeschoben wird. ${ }^{14}$ In keinem Fall ist es aber hinreichend, dass das Leben einer Person verlängert wird, indem die tödliche Wirkung einer Lebensgefahr nur um kurze Zeit aufgeschoben wird. Es gilt also nicht, dass in jedem Fall einer Lebensverlängerung auch eine Lebensrettung vorliegt. Dann kann es aber auch nicht sein, dass Lebensrettung und Lebensverlängerung (in deskriptiver Hinsicht) das Gleiche sind,

\footnotetext{
13 Es könnte auch sein, dass es gar keine objektive Antwort hierauf gibt, da der Begriff der Lebensrettung in Bezug auf solche Fälle keine eindeutigen Anwendungsregeln haben und damit unterbestimmt sein könnte. Das ist dadurch plausibel, dass Fälle, in denen Leben um sehr lange Zeit verlängert werden, ohne dass die drohende Todesgefahr beseitigt wird, sehr ungewöhnlich sind. Eine begriffliche Unterbestimmtheit des Begriffs der Lebensrettung wird auch von Sebastian Knell (2011: 12) in Erwägung gezogen.

14 In diesem Fall würde es im Sinne der Bündeltheorie von Begriffen (s. Baumann 2002: 101-2) also zwei Bündel von Eigenschaften geben, die jeweils hinreichend, aber nicht notwendig für das Vorliegen einer Lebensrettung sind, wobei das eine Bündel aus $<$ Lebensverlängerung durch vollständige Abwendung einer Todesgefahr $>$ und das andere Bündel aus <Lebensverlängerung durch langen Aufschub der tödlichen Wirkung der Todesgefahr> bestünden.
} 
wie die Prämisse $\mathrm{H} 1$ besagt. H1 stellt sich damit als falsch und Harris' Argument entsprechend als unschlüssig heraus.

\section{Zwei alternative Argumente für eine prima facie Pflicht zur direkten Lebensverlängerung}

Ich habe im vorherigen Abschnitt dafür argumentiert, dass die Prämisse H1 von Harris' Argument nicht wahr ist, da die Verlängerung eines Lebens zwar notwendig, nicht aber hinreichend für eine Lebensrettung ist, da für letztere zudem notwendig ist, dass die Lebensverlängerung durch die vollständige Abwendung einer Todesgefahr bewirkt wurde oder aber zumindest durch den Aufschub der tödlichen Wirkung einer Todesgefahr um lange Zeit. Aus der Prämisse H1 wird in Harris' Argument zusammen mit $\mathrm{H} 2$ auf die Zwischenkonklusion $\mathrm{K} 1$ geschlossen, welche besagt, dass Lebensverlängerung und Lebensrettung moralisch äquivalent sind, wovon wiederum zusammen mit anderen Prämissen die endgültige Konklusion K3 abgeleitet wird, dass wir - von Ausnahmefällen abgesehen - prima facie zur direkten Lebensverlängerung verpflichtet sind. Wenn $\mathrm{H} 1$ falsch ist, ist das Argument daher unschlüssig. Daraus, dass die Prämisse H1 falsch ist, folgt aber natürlich weder, dass K1 falsch ist, noch, dass $\mathrm{K} 3$ falsch ist: Es kann trotzdem sein, dass Lebensverlängerung und Lebensrettung moralisch äquivalent sind und wir eine prima facie Pflicht zur direkten Lebensverlängerung haben. Ob dies der Fall ist, werde ich im Folgenden auf Basis von Harris' Argument und meiner daran geäußerten Kritik untersuchen.

Meine Auseinandersetzung mit dem Begriff der Lebensrettung hat zwar ergeben, dass ein Leben zu retten nicht das Gleiche ist, wie ein Leben zu verlängern; auf der anderen Seite hat sich aber gezeigt, dass eine Lebensverlängerung immerhin eine notwendige Bedingung für eine Lebensrettung ist und nur eine weitere Bedingung erfüllt werden muss, damit eine Lebensrettung vorliegt. Es gibt nun zwei naheliegende Möglichkeiten, wie alternativ begründet werden könnte, dass wir eine prima facie Pflicht zur direkten Lebensverlängerung haben: Zum einen könnte es sein, dass zwar nicht Lebensverlängerung per se, wohl aber direkte Lebensverlängerung oder zumindest bestimmte technologische Umsetzungen derselben die (bzw. eine) zusätzliche Bedingung erfüllen, die für das Vorliegen einer Lebensrettung notwendig ist, sodass es sich bei diesen um Lebensrettungen handelt. Dann wären zwar nicht - wie K1 besagt - Lebensverlängerung und Lebensrettung moralisch äquivalent, wohl aber direkte Lebensverlängerung (bzw. bestimmte Umsetzungen derselben) und Lebensrettung, sodass wir doch - wie K3 besagt - prima facie zur direkten Lebensverlängerung verpflichtet wären (bzw. zu bestimmten Umsetzungen derselben).

Zum anderen könnte sich sogar herausstellen, dass die von K1 behauptete moralische Äquivalenz von (bloßer) Lebensverlängerung und Lebensrettung zutrifft, sodass davon - wie in Harris' Argument - zusammen mit H3 und H4 die endgültige Konklusion K3 abgeleitet werden kann. Das wäre dann der Fall, wenn die zusätzliche notwendige Bedingung für das Vorliegen einer Lebensrettung moralisch irrelevant oder zumindest nicht so relevant ist, dass ohne sie der Status einer prima facie Pflicht verloren geht. Ein solches Argument wäre analog zu Singers (1972) 
Analogie-Argument dafür, dass wir verpflichtet sind, Menschen in extremer Armut durch Geldspenden zu helfen. Er behauptet, dass eine solche Handlung moralisch äquivalent zur Rettung eines ertrinkenden Kindes aus einem nahegelegenen Teich ist. In diesem Fall ist es offensichtlich, dass zwei (in deskriptiver Hinsicht) unterschiedliche Handlungen vorliegen. Trotzdem argumentiert Singer dafür, dass die beiden Handlungen moralisch äquivalent sind, indem er behauptet, dass alle Unterschiede zwischen den beiden Handlungen - wie zum Beispiel die Distanz zur geretteten Person - moralisch irrelevant sind. Wenn nun alle Unterschiede moralisch irrelevant sind, so folgt, dass die beiden Handlungen in jeder moralisch relevanten Hinsicht gleich und damit moralisch äquivalent sind.

Ich werde im Folgenden prüfen, ob die beiden skizzierten Argumentationsgänge im Fall der direkten Lebensverlängerung erfolgreich sind.

\subsection{Direkte Lebensverlängerung und Lebensrettung}

Ich beginne mit der Frage, ob es sich bei (bestimmten Umsetzungen von) direkten Lebensverlängerungen - im Gegensatz zur (bloßen) Lebensverlängerung - um Lebensrettungen handelt, indem diese eine zusätzliche Bedingung erfüllen, die für das Vorliegen einer Lebensrettung nötig ist. Meine Begriffsanalyse hat ergeben, dass eine solche zusätzliche Bedingung darin besteht, dass eine bestehende Todesgefahr vollständig abgewendet wird, und eine alternative Bedingung darin bestehen könnte, dass die tödliche Wirkung einer Todesgefahr um lange Zeit aufgeschoben wird. Da offengeblieben ist, ob letztere alternative Bedingung tatsächlich hinreicht, kann nur dann mit Sicherheit gesagt werden, dass es sich bei direkten Lebensverlängerungen um Lebensrettungen handelt, wenn gezeigt werden kann, dass die erstere Bedingung erfüllt ist, also eine bestehende Todesgefahr vollständig abgewendet wird.

Ich denke, dass durch eine direkte Lebensverlängerung eine Todesgefahr zumindest abgewendet werden kann: Die direkte Lebensverlängerung verlängert Leben (unter anderem) dadurch, dass verhindert wird, dass Menschen zu einem bestimmten Zeitpunkt an altersbedingten Krankheiten sterben (s. Ehni 2013: 255). Es kann natürlich sein, dass der Zeitpunkt des Eintritts dieser Krankheiten nur aufgeschoben wird; es kann aber auch sein, dass diese Krankheiten gar nicht mehr auftreten und die Person an einer anderen Todesursache stirbt, sodass die altersbedingten Krankheiten vollständig abgewendet werden. Tatsächlich scheint es sogar recht wahrscheinlich, dass zumindest eine altersbedingte Krankheit vollständig abgewendet wird, sodass durch Maßnahmen der direkten Lebensverlängerung mit einer substanziellen Wahrscheinlichkeit Leben gerettet werden. ${ }^{15}$ Der einzig moralisch zulässige Umgang mit

\footnotetext{
15 Wie hoch diese Wahrscheinlichkeit ist, hängt von der konkreten technologischen Realisierung der direkten Lebensverlängerung ab. Ich setzte hier voraus, dass die direkte Lebensverlängerung effektiv genug ist, um das Leben einer Person um viele Jahre zu verlängern.
} 
dieser epistemischen Unsicherheit besteht aber darin, die Maßnahmen vorzunehmen, sodass wir prima facie zur direkten Lebensverlängerung verpflichtet sind. ${ }^{16}$

Ein naheliegender Einwand ist, dass es für eine Lebensrettung nicht hinreicht, einzelne altersbedingte Krankheiten abzuwenden, da zur lebensbedrohlichen Situation, die abgewendet werden muss, auch das hohe Alter selbst oder alle altersbedingten Krankheiten gehören. Solange die direkte Lebensverlängerung den Alterungsprozess nur verlangsamt und nicht verhindert, werden aber weder das hohe Alter noch alle altersbedingten Krankheiten vollständig abgewendet. Dieser Einwand hat zwar eine gewisse anfängliche Plausibilität, ist aber nicht schlüssig. Wenn es notwendig wäre, auch das hohe Alter oder alle anderen altersbedingten Krankheiten abzuwenden, würde nämlich bei keiner Heilung einer einzelnen tödlichen altersbedingten Krankheit eine Lebensrettung vorliegen. Viele dieser Fälle - wie z. B. wenn eine alte Person von Krebs geheilt wird - sind aber paradigmatische Fälle von Lebensrettungen. Also handelt es sich auch dann bei der Abwendung einer altersbedingten Krankheit um eine Lebensrettung, wenn dadurch weder das hohe Alter noch alle anderen altersbedingten Krankheiten abgewendet werden. Die erste Argumentationsstrategie für eine prima facie Pflicht zur direkten Lebensverlängerung scheint daher erfolgreich zu sein.

\subsection{Eine moralische Analogie zur Lebensrettung}

Als nächstes werde ich den zweiten oben vorgeschlagenen Argumentationsgang prüfen, nach dem Lebensrettung und Lebensverlängerung trotz ihrer (deskriptiven) Verschiedenheit moralisch äquivalent sind, sodass sich eine moralische Analogie zwischen den beiden ergibt. Wie oben erläutert, muss dafür eruiert werden, ob die zusätzliche Bedingung für Lebensrettung, dass die Lebensverlängerung durch die vollständige Abwendung einer Todesgefahr bewirkt wurde, moralisch relevant ist. Dies kann getestet werden, indem man sich einen Hilfskonflikt vorstellt, in dem das Leben von zwei Personen bedroht ist und man nur das Leben einer Person verlängern kann, wobei man über die weitere (und sonst keine weitere) Information verfügt, dass man die Todesgefahr, die das Leben der einen Person bedroht, vollständig abwenden kann, während es ungewiss ist, ob man die Lebensverlängerung der anderen Person durch die vollständige Abwendung einer Todesgefahr oder durch eine bloße Aufschiebung der tödlichen Wirkung einer Todesgefahr bewirkt. Wenn einem keine weitere Informationen gegeben sind, sollte man sich für die Person entscheiden, von der man weiß, dass man die Todesgefahr nicht nur aufschieben, sondern vollständig abwenden kann. Dann muss es aber moralisch relevant sein,

\footnotetext{
16 Nicht jede Unsicherheit darüber, ob eine Maßnahme eine Lebensrettung bewirkt, verpflichtet zu dieser Maßnahme. Entscheidend ist, dass durch die Maßnahme mit einer hinreichenden Wahrscheinlichkeit eine Lebensrettung bewirkt wird. Dass aber keine vollkommene Sicherheit darüber bestehen muss, dass eine Maßnahme eine Lebensrettung bewirken würde, um diese Maßnahme verpflichtend zu machen, kann man sich daran klar machen, dass selbst in idealtypischen Lebensrettungen solch eine Sicherheit (meistens) nicht besteht. Siehe dazu die bereits diskutierten Beispiele: Vielleicht hätte sich der Nichtschwimmer doch noch aus dem Wasser kämpfen können, und die vergiftete Person hätte ihre Vergiftung möglicherweise auch ohne Antidot überlebt.
} 
ob eine Lebensverlängerung durch die vollständige Abwendung einer Todesgefahr bewirkt wird.

Ein Grund für die moralische Relevanz scheint darin zu bestehen, dass zu erwarten ist, dass man das Leben einer Person um mehr Zeit verlängert, wenn man eine Todesgefahr, die ihr Leben bedroht, vollständig abwendet, statt nur aufzuschieben. Hinzu kommt, dass im Fall der vollständigen Abwendung der Todesgefahr auch die Qualität der zusätzlichen Lebenszeit (pro Einheit Lebenszeit) in Erwartung höher ist, da eine nur aufgeschobene Todesgefahr - wie zum Beispiel im Fall von tödlichen Verletzungen - bis zu ihrer tödlichen Wirkung starkes Leid verursachen kann. Wenn sich die moralische Relevanz der vollständigen Abwendung einer Todesgefahr auf diese beiden Faktoren zurückführen lässt, so sollten Lebensverlängerung und Lebensrettung moralisch äquivalent sein, wenn diese beiden Faktoren in beiden Fällen gleich ausgeprägt sind. Dies kann erneut durch die intuitive Bewertung eines Hilfskonfliktes geprüft werden, in dem man nur das Leben einer von zwei Personen verlängern kann, wobei nur die folgenden Informationen gegeben sind: Das Leben der einen Person kann man verlängern, indem man die Todesgefahr, die sie bedroht, vollständig abwendet, wobei sie aber (mit Sicherheit) nach X Jahren an einer anderen Todesursache sterben wird. Die andere Person wird nicht zusätzlich von einer anderen Todesursache bedroht; ihr Leben kann man aber nur verlängern, indem man die tödliche Wirkung ihrer Todesgefahr um X Jahre aufschiebt, wobei sich diese Todesgefahr bis zu ihrer tödlichen Wirkung nicht auf ihre Lebensqualität auswirken wird. In diesem Fall scheint es moralisch irrelevant zu sein, ob man sich für die Lebensverlängerung der einen oder der anderen Person entscheidet, obgleich es sich im ersten Fall um eine Lebensrettung und im zweiten ausschließlich um eine Lebensverlängerung handeln würde. Während keine moralische Äquivalenz zwischen Lebensrettung und Lebensverlängerung vorliegt, besteht demnach moralische Äquivalenz zwischen einer Lebensrettung, welche das Leben der geretteten Person um X Zeiteinheiten verlängert, und einer (bloßen) Lebensverlängerung um X Zeiteinheiten, wenn die Qualität der zusätzlichen Lebensjahre in beiden Fällen gleich groß ist. Hieraus folgt, dass wir zur Lebensverlängerung um X Zeiteinheiten der Qualität Q prima facie verpflichtet sind, wenn wir zur Lebensrettung, welche eine Lebensverlängerung um X Zeiteinheiten der Qualität Q bewirkt, prima facie verpflichtet sind.

Bei welcher Lebensqualität Q und wie vielen Zeiteinheiten $\mathrm{X}$ sind wir zur Lebensrettung verpflichtet? Wie in der Diskussion der Prämisse H3 von Harris' Argument erläutert, sind dafür eine mehr als minimale Verlängerung der Lebenszeit bei einer akzeptablen bzw. lebenswerten Lebensqualität hinreichend, wenn die gerettete Person zudem gerettet werden will. ${ }^{17}$ Es folgt, dass wir auch zu einer Lebensverlängerung um eine mehr als minimale Zeit bei einer lebenswerten Lebensqualität prima facie verpflichtet sind, wenn die Person, deren Leben verlängert wird,

\footnotetext{
17 Es konnte in diesem Aufsatz nicht geklärt werden, welche dieser Bedingungen für das Vorliegen einer prima facie Pflicht zur Lebensrettung notwendig sind. Deshalb habe ich mich hier damit begnügt, dafür zu argumentieren, dass wir eine prima facie Pflicht zur direkten Lebensverlängerung haben, wenn alle drei Bedingungen erfüllt sind. Es kann aber gut sein, dass auch dann eine prima facie Pflicht besteht, wenn nicht alle dieser Bedingungen simultan erfüllt sind.
} 
diese Lebensverlängerung will. Zusammen mit der bereits bestätigten Prämisse H4, dass wir zur direkten Lebensverlängerung prima facie verpflichtet sind, wenn wir zur Lebensverlängerung prima facie verpflichtet sind, folgt dann, dass wir unter diesen Umständen auch zur direkten Lebensverlängerung prima facie verpflichtet sind.

\section{Fazit}

Ich habe mich in diesem Aufsatz mit der Frage auseinandergesetzt, welchen moralischen Status die direkte Lebensverlängerung hat. Im Fokus dieser Auseinandersetzung stand Harris' Argument dafür, dass wir eine prima facie Pflicht zur direkten Lebensverlängerung haben, die moralisch äquivalent zu der prima facie Pflicht zur Lebensrettung ist. Meine Untersuchung dieses Arguments hat ergeben, dass es nicht schlüssig ist, da die begriffliche Prämisse, nach der Lebensrettung und Lebensverlängerung das Gleiche sind, falsch ist. Zwar konnte Wittwers Kritik an dieser Prämisse zurückgewiesen werden; ein begrifflicher Unterschied besteht jedoch darin, dass es für eine Lebensrettung notwendig ist, dass die Todesgefahr, die das Leben der geretteten Person bedroht hat, vollständig abgewendet (oder aber zumindest um sehr lange Zeit aufgeschoben) wurde. Auf Basis dieser Begriffsanalyse konnten dann aber zwei alternative Argumente für eine prima facie Pflicht zur direkten Lebensverlängerung konstruiert werden, die auf Harris' Überlegungen aufbauen. Im ersten Argument wurde argumentiert, dass die direkte Lebensverlängerung - im Gegensatz zur (bloßen) Lebensverlängerung - die zusätzliche notwendige Bedingung für eine Lebensrettung mit einer substantiellen Wahrscheinlichkeit erfüllt, sodass es gut sein kann, dass durch sie Leben gerettet werden würden. Dass dies wahrscheinlich ist, ist aber bereits hinreichend dafür, dass wir prima facie verpflichtet wären, Maßnahmen der direkten Lebensverlängerung durchzuführen. Im zweiten Argument wurde argumentiert, dass die zusätzliche notwendige Bedingung für eine Lebensrettung nur insofern moralisch relevant ist, als sie indiziert, dass das Leben der geretteten Person um mehr und bessere Lebenszeit verbessert wird als bei einer bloßen Lebensverlängerung, sodass zwar nicht Lebensrettung und Lebensverlängerung per se, wohl aber Lebensrettung und Lebensverlängerung bei gleich langer und gleich guter zusätzlicher Lebenszeit moralisch äquivalent sind. Auf Basis von Harris' restlichem Argument konnte dann abgeleitet werden, dass wir - von Ausnahmefällen abgesehen - eine prima facie Pflicht zur direkten Lebensverlängerung haben.

$\mathrm{Ob}$ wir in bestimmten biotechnologischen Zukunftsszenarien auch all things considered zur direkten Lebensverlängerung verpflichtet wären, wurde in diesem Aufsatz nicht beantwortet. Dafür müssten zusätzlich die in der Einführung erwähnten populationsethischen und gerechtigkeitstheoretischen sowie weitere moralische Gesichtspunkte berücksichtigt und mit der prima facie Pflicht zur direkten Lebensverlängerung abgewogen werden. Während es eine offene Frage ist, welcher moralische Status der direkten Lebensverlängerung sich aus solch einer Abwägung ergäbe, zeigen die Resultate dieses Aufsatzes, dass die hier nicht diskutierten moralischen Gesichtspunkte insgesamt starke Gründe gegen die direkte Lebensverlängerung bilden müssten, um die Unterlassung einer direkten Lebensverlängerung moralisch zulässig (oder sogar verpflichtend) zu machen. In der Tat müssten dafür die anderen 
moralischen Gesichtspunkte so starke Gründe gegen die direkte Lebensverlängerung bilden, wie nötig wären, um die Unterlassung einer Lebensrettung, welche das gerettete Leben um die gleiche Dauer und Qualität verlängern würde, moralisch zulässig (oder sogar verpflichtend) zu machen.

Danksagung Für Anregungen und hilfreiche Kommentare danke ich Sebastian Knell, Niklas Kurzböck und Achim Lohmar.

Funding Open Access funding enabled and organized by Projekt DEAL.

Open Access Dieser Artikel wird unter der Creative Commons Namensnennung 4.0 International Lizenz veröffentlicht, welche die Nutzung, Vervielfältigung, Bearbeitung, Verbreitung und Wiedergabe in jeglichem Medium und Format erlaubt, sofern Sie den/die ursprünglichen Autor(en) und die Quelle ordnungsgemäß nennen, einen Link zur Creative Commons Lizenz beifügen und angeben, ob Änderungen vorgenommen wurden.

Die in diesem Artikel enthaltenen Bilder und sonstiges Drittmaterial unterliegen ebenfalls der genannten Creative Commons Lizenz, sofern sich aus der Abbildungslegende nichts anderes ergibt. Sofern das betreffende Material nicht unter der genannten Creative Commons Lizenz steht und die betreffende Handlung nicht nach gesetzlichen Vorschriften erlaubt ist, ist für die oben aufgeführten Weiterverwendungen des Materials die Einwilligung des jeweiligen Rechteinhabers einzuholen.

Weitere Details zur Lizenz entnehmen Sie bitte der Lizenzinformation auf http://creativecommons.org/ licenses/by/4.0/deed.de.

\section{Literatur}

Barzilai, Nir, Crandall, Jill P., Kritchevsky, Stephen B., \& Espeland, Mark A. 2016. Metformin as a tool to target aging. Cell metabolism 23(6):1060-1065.

Baumann, Peter. 2002. Erkenntnistheorie. Stuttgart: J.B. Metzler.

Bostrom, Nick. 2005. The fable of the dragon tyrant. Journal of medical ethics 31(5): 273-277.

Caplan, Arthur L. 2004. An unnatural process: Why it is not inherently wrong to seek a cure for aging. In The Fountain of Youth: Cultural Scientific and Ethical Perspectives on a Biomedical Goal, ed. S. G. Post \& R. H. Binstock, 271-286. Oxford: Oxford University Press.

Chapman, Audrey R. 2004 The social and justice implications of extending the human life span. In The Fountain of Youth: Cultural Scientific and Ethical Perspectives on a Biomedical Goal, ed. S. G. Post \& R. H. Binstock, 340-362. Oxford: Oxford University Press.

Ehni, Hans-Jörg. 2013. Ethik der Biogerontologie. Wiesbaden: Springer VS.

Farrelly, Colin. 2008. Aging research: priorities and aggregation. Public Health Ethics 1 (3):258-267.

Freitas Jr., Robert A. 2009. Nanomedizin. Die Suche nach unfallbegrenzten Lebensspannen. In Länger leben? Philosophische und biowissenschaftliche Perspektiven, Hrsg. Sebastian Knell \& Marcel Weber, 63-77. Frankfurt am Main: Suhrkamp.

Gems, David. 2009. Eine Revolution des Alterns. Die neue Biogerontologie und ihre Implikationen. In Länger leben? Philosophische und biowissenschaftliche Perspektiven, Hrsg. Sebastian Knell \& Marcel Weber, 25-46. Frankfurt am Main: Suhrkamp.

Harris, John. 2007. Enhancing Evolution: The Ethical Case for Making Better People. Princeton: Princeton University Press.

Kass, Leon R. 2003. Ageless bodies, happy souls: biotechnology and the pursuit of perfection. The New Atlantis 1:9-28.

Knell, Sebastian. 2011. Anti-Aging, Leben-Retten und Gerechtigkeit. Reflexionen zur Moral der Lebensverlängerung. Jahrbuch für Wissenschaft und Ethik 16(1):5-40.

Lutz, Matthew und James Lenman. 2018. Moral Naturalism. The Stanford Encyclopedia of Philosophy (Fall 2018 Edition), ed. Edward N. Zalta. https://plato.stanford.edu/archives/fall2018/entries/ naturalism-moral/. 
Rose, Michael R. 2009. Realismus in Sachen Anti-Aging. In Länger leben? Philosophische und biowissenschaftliche Perspektiven, Hrsg. Sebastian Knell \& Marcel Weber, 46-63. Frankfurt am Main: Suhrkamp.

Schloendorn, John. 2006. Making the case for human life extension: personal arguments. Bioethics 20(4):191-202.

Singer, Peter. 1972. Famine, Affluence, and Morality. Philosophy and Public Affairs 1(3):229-243.

Singer, Peter. 2009. Die Erforschung des Alterns und die Interessen gegenwärtiger Individuen, zukünftiger Individuen sowie der Spezies. In Länger leben? Philosophische und biowissenschaftliche Perspektiven, Hrsg. Sebastian Knell \& Marcel Weber, 152-174. Frankfurt am Main: Suhrkamp.

Williams, Bernard. 1973. The Makropulos Case: Reflections on the Tedium of Immortality. In Problems of the Self, 81-100. Cambridge: Cambridge University Press.

Wittwer, Héctor. 2009. Warum die direkte technische Lebensverlängerung nicht moralisch geboten ist. In Länger leben? Philosophische und biowissenschaftliche Perspektiven, Hrsg. Sebastian Knell \& Marcel Weber, 210-235. Frankfurt am Main: Suhrkamp. 